
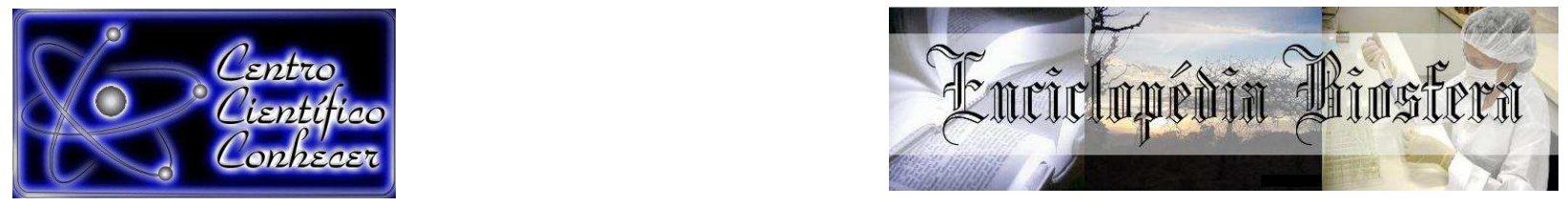

\title{
ESTRUTURA ARBUSTIVO-ARBÓREA DE CERRADO RUPESTRE DA REGIÃO SUDOESTE DO ESTADO DE GOIÁS, BRASIL
}

Benaya Nogueira Leles ${ }^{1}$ e Vania Sardinha dos Santos Diniz ${ }^{2}$

1. Mestre em Ecologia de Ecótonos, Universidade Federal do Tocantins.

2. Professora Doutora do Ensino Básico, Técnico e Tecnológico do Instituto Federal Goiano, Campus Iporá. Av. Oeste, s/n, saída para Piranhas, Iporá, GO. CEP: 76200-

000. Caixa Postal: 51._(vania.diniz@ifgoiano.edu.br)

Recebido em: 02/10/2017 - Aprovado em: 21/11/2017 - Publicado em: 05/12/2017 DOI: 10.18677/EnciBio_2017B83

\begin{abstract}
RESUMO
cerrado rupestre possui grande riqueza de espécies endêmicas e raras, mesmo assim apresenta uma área cada vez mais reduzida, principalmente devido à ação antrópica. O presente trabalho realizou o levantamento da estrutura arbustivoarbórea em uma área remanescente de cerrado rupestre, onde a visitação humana é intensa devido ao ecoturismo. Foram utilizadas 10 parcelas permanentes de $20 \times 50$ m (1 ha), sendo amostrados todos os indivíduos com diâmetro igual ou superior a $5,0 \mathrm{~cm}$ a $30 \mathrm{~cm}$ do solo. Foram calculados os valores absolutos e relativos de densidade, dominância, frequência, valor de importância e área basal, o índice de diversidade de Shannon ( $\mathrm{H}^{\prime}$ ) e o de uniformidade de Pielou (J). A densidade total da área foi de 543 indivíduos.ha-1 distribuídos em 58 espécies, 46 gêneros e 28 famílias. A área basal foi de $4,6 \mathrm{~m}^{2}$. As famílias com maior número de espécies foram: Fabaceae (8) e Vochysiaceae (7). Quinze famílias foram representadas por uma única espécie e 16 espécies apresentaram apenas um indivíduo. Qualea parviflora, Davilla elliptica, Sclerolobium paniculatum var. subvelutinum, Miconia ferruginata, Caryocar brasiliense apresentaram os maiores índices de valor de importância. Foi registrada pela primeira vez no cerrado rupestre do estado de Goiás a espécie Hirtella gracilipes, típica da formação de Cerradão. O índice de Shannon $\left(H^{\prime}\right)$ foi de 1,51 e o de uniformidade de Pielou $(\mathrm{J})$ igual a 0,37 , valores baixos quando comparados com outras áreas de cerrado rupestre, indicando que esta área pode estar sofrendo ações de degradação provocada principalmente pela intensa visitação humana.
\end{abstract}

PALAVRAS-CHAVE: Afloramentos rochosos, flora lenhosa. Fitossociologia. 


\title{
STRUCTURE OF THE TREE AND SHRUB VEGETATION OF ROCKY SOILS OF SOUTHWEST REGION OF THE STATE OF GOIÁS, BRAZIL.
}

\begin{abstract}
The rocky soils area shelters a large number of endemic and rare species, even though they present an increasingly smaller area, mainly due to anthropic action. The present work carried out the survey of the tree and shrub vegetation structure in a remnant area of rocky soil, where the human visitation is intense due to ecotourism. In the area ten permanent plots of $20 \times 50 \mathrm{~m}$ (1 ha) were allocated, were registered with trunk diameters equal to or greater than $5 \mathrm{~cm}$ in height of $30 \mathrm{~cm}$ of soil. The phytossociological analysis was based on the absolute and relative parameters of density, dominance, frequency, importance values and basal area values. We calculated the diversity Shannon index $\left(\mathrm{H}^{\prime}\right)$ and uniformity of Pielou index (J'). The overall density area was 543 individual. ha ${ }^{-1}$ in 58 species, 46 genera and 28 families. The basal area of $4.6 \mathrm{~m}^{2}$. The Fabaceae had the highest number of species (8), followed by Vochysiaceae (7). Fifteen other families were represented by a single species and 16 species had only one individual. Qualea parviflora, Davilla elliptica, Sclerolobium paniculatum var. subvelutinum, Miconia ferruginata and Caryocar brasiliense showed the highest levels of importance value. The species Hirtella gracilipes, typical of "Cerradão" formation, was recorded for the first time in the rock soil of the state of Goiás.The Shannon index $\left(\mathrm{H}^{\prime}\right)$ was 1.51 and uniformity of Pielou index (J') was 0,37 , which were low compared to other areas of rocky soils. .

KEYWORDS: fitossociology, rocky soils, woody flora.
\end{abstract}

\section{INTRODUÇÃO}

Um gradiente de fisionomias, sendo: campestres, savânicas e florestais constituem o domínio Cerrado (RIBEIRO; WALTER, 2008). Entre as formações savânicas, o cerrado rupestre é caracterizado pela presença de afloramentos rochosos, vegetação rasteira e ambientes abertos (RIBEIRO; WALTER; 2008; ALVES et al., 2014). A flora é semelhante ao cerrado sensu stricto, onde as mudanças nos parâmetros florísticos não são significativas (GOMES et al., 2016), no entanto, a presença das rochas modifica a composição florística, pois a distribuição das espécies é diferente entre os substratos (MOTA et al., 2014, TORRES et al., 2017).

As áreas de cerrado rupestre apresentam variada biodiversidade principalmente por possuírem diversos microhabitats, como fendas, fissuras e rochas expostas (SILVA, 2016) abrigando grande número de espécies endêmicas, raras e ameaçadas (SILVA; GERMANO, 2013; VERÇOZA; BASTOS, 2013; ANTUNES et al., 2013). Além de serem fontes para a descoberta de novas espécies, também são importantes para a disseminação de propágulos para áreas vizinhas do Cerrado (FREITAS et al., 2016).

Inventários fitossociológicos são importantes, pois fornecem o histórico de como as plantas se organizam no espaço podendo ser utilizados para o planejamento de políticas de conservação (MELO et al., 2013). Isso reforça a necessidade do aumento do número de estudos em afloramentos rochosos que proponham políticas de manejo para o ecossistema, fornecendo subsídios para conservação das espécies (SILVA, 2016).

Embora os estudos em áreas de afloramentos rochosos tenham aumentado 
nos últimos anos, estes ainda são insuficientes, principalmente devido a elevada taxa de destruição que essas formações vêm sofrendo ao longo do tempo principalmente pelas ações antrópicas (SILVA, 2016). As crescentes ameaças às formações rupestres comprometem os serviços ecossistêmicos e faz-se necessária a implantação de estratégias de conservação e restauração mais eficazes dessas áreas (SILVEIRA et al., 2016).

Com o objetivo de ampliar o conhecimento sobre a vegetação do Cerrado, principalmente sobre áreas de afloramentos rochosos, foi realizado o levantamento fitossociológico do cerrado rupestre do Salto de São Domingos, município de Piranhas, região Sudoeste do Estado de Goiás.

\section{MATERIAL E MÉTODOS}

\section{Área de estudo}

O presente estudo foi realizado no cerrado rupestre próximo ao Salto de São Domingos, que se localiza no município de Piranhas, na região Sudoeste do Estado de Goiás, (16²5’37'S e 5049’20"W). O município apresenta 11.266 habitantes, de acordo com o censo de 2010, área total de $2.048 \mathrm{Km}^{2}$ e altitude média de $389 \mathrm{~m}$ (IBGE, 2017). O clima predominante na região é tipo Aw da classificação de Köppen, típicos de savanas tropicais.

O córrego de São Domingos é bastante frequentado pela população dos municípios de Piranhas, Caiapônia e região do Oeste e Sudoeste goiano, a vegetação apresenta tanto áreas de savana como de matas. O Salto de São Domingos é a segunda maior queda d'água do Estado de Goiás, com $96 \mathrm{~m}$ de altura e de grande beleza cênica é o local mais visitado do córrego São Domingos (SANTOS, 2001). O Salto está localizado a $43 \mathrm{Km}$ da cidade de Piranhas, o acesso é feito a partir da sede do município pela GO-188, sentido Doverlândia, segundo informação dos guias turísticos locais. Apesar de apresentar potencial turístico, não existe um plano de manejo para a conservação dos recursos naturais dessa área e nenhum registro do levantamento da flora local.

\section{Amostragem da vegetação}

Para a amostragem foi utilizado o método de parcelas. Foram alocadas 10 parcelas de área fixa de $20 \times 50 \mathrm{~m}$, totalizando uma área amostral de um ha, conforme recomendado para levantamento da vegetação nas formações savânicas do Cerrado (FELFILI et al., 2005).

No levantamento foram incluídos todos os indivíduos com diâmetro do caule igual ou superior a $5 \mathrm{~cm}$ a $30 \mathrm{~cm}$ do nível do solo (FELFILI et al., 2005). Todos os indivíduos dentro do critério de inclusão tiveram os diâmetros medidos com suta metálica e altura máxima da copa medida com trena. A identificação dos indivíduos foi realizada in loco utilizando literatura especializada e o sistema de classificação do Angiosperm Phylogeny Group (APG III, 2009) e os nomes científicos foram atualizados utilizando a Lista de Espécies da Flora do Brasil (FLORA DO BRASIL 2020, 2016).

\section{Análise fitossociológica e medidas de diversidade}

A fitossociologia foi feita a partir da obtenção, para cada espécie, dos seguintes parâmetros: densidade absoluta, densidade relativa (DR), área basal $(A B)$, frequência absoluta (FA), frequência relativa $(F R)$, dominância relativa $(D o R)$, valor 
de importância (VI) (MULLER-DUMBOIS; ELLEMBERG, 1974). Foi feita a avaliação da distribuição vertical e horizontal, através das medidas da altura máxima e diâmetro do caule de cada indivíduo amostrado. Para avaliar a diversidade foi utilizado o índice de diversidade de Shannon-Wiener (H') (MAGURRAN, 1988) e para separar a equabilidade foi calculado o índice de uniformidade de Pielou (J') (PIELOU, 1975).

\section{RESULTADOS E DISCUSSÃO}

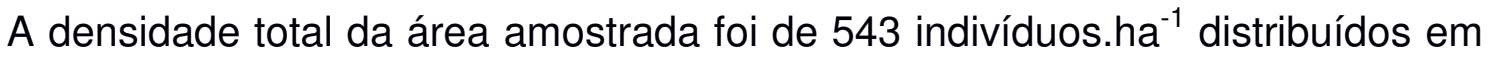
58 espécies, 46 gêneros e 28 famílias. A área basal total foi de 4,6 $\mathrm{m}^{2}$. As famílias que mais se destacaram em número de espécie foram: Fabaceae (8); Vochysiaceae (7); Apocynaceae (4), Malpighiaceae (4), Melastomataceae (4) e Rubiaceae (4), representando $55,3 \%$ do total das amostras. Destas, quatro famílias também tiveram maior representação em número de indivíduos: Vochysiaceae (124), Fabaceae (99), Melastomataceae (74) e Malpighiaceae (62) (Tabela 1).

TABELA 1 - Espécies, nas respectivas famílias, densidade (D); densidade relativa (DR\%), frequência relativa (FR\%); dominância relativa (DoR\%) e valor de importância (VI) correspondente à amostragem da vegetação em área de cerrado rupestre no Salto de São Domingos, município de Piranhas, Goiás, Brasil.

\begin{tabular}{lrrrrr}
\hline Espécies (Família) & NI & DR & FR & DoR & VI \\
\hline Qualea parviflora Mart (Vochysiaceae) & 44 & 8,10 & 4,24 & 11,30 & 23,65 \\
Davilla elliptica A.St.-Hil. (Dilleniaceae) & 53 & 9,76 & 6,06 & 5,45 & 21,27 \\
Sclerolobium paniculatum Vogel var subvelutinum & & & & & \\
Benth (Fabaceae) & 41 & 7,55 & 2,42 & 9,72 & 19,70 \\
Miconia ferruginata DC. (Melastomataceae) & 37 & 6,81 & 4,85 & 4,33 & 16,00 \\
Caryocar brasiliense Cambess. (Caryocaraceae) & 18 & 3,31 & 4,24 & 6,05 & 13,61 \\
Vochysia thyrsoidea Pohl. (Vochysiaceae) & 30 & 5,52 & 3,03 & 3,45 & 12,01 \\
Andira cujabensis Benth. (Fabaceae) & 20 & 3,68 & 2,42 & 4,78 & 10,88 \\
Qualea grandiflora Mart. (Vochysiaceae) & 16 & 2,95 & 2,42 & 3,76 & 9,13 \\
Xylopia aromatica (Lam.) Mart. (Annonaceae) & 13 & 2,39 & 4,24 & 2,17 & 8,81 \\
Byrsonima sp. (Malpighiaceae) & 23 & 4,24 & 2,42 & 2,01 & 8,67 \\
Hymenae stigonocarpa Mart ex Hayne (Fabaceae) & 12 & 2,21 & 3,03 & 2,83 & 8,07 \\
Salvertia convallariodora A. St.-Hil. (Vochysiaceae) & 14 & 2,58 & 2,42 & 2,45 & 7,46 \\
Emmotum nitens (Benth.) Miers (Icacinaceae) & 6 & 1,10 & 1,21 & 5,06 & 7,38 \\
Kielmeyera coriacea Mart \& Zucc. & & & & & \\
(Calophyllaceae) & 11 & 2,03 & 3,03 & 2,29 & 7,35 \\
Vochysia rufa Mart. (Vochysiaceae) & 13 & 2,39 & 2,42 & 2,33 & 7,14 \\
Byrsonima pachyphylla A. Juss. (Malpighiaceae) & 17 & 3,13 & 2,42 & 1,08 & 6,64 \\
Byrsonima verbascifolia (L.) DC. (Malpighiaceae) & 16 & 2,95 & 2,42 & 1,18 & 6,55 \\
Miconia burchellii Triana (Melastomataceae) & 17 & 3,13 & 1,82 & 1,48 & 6,43 \\
Bowdichia virgilioides Kunth (Fabaceae) & 9 & 1,66 & 1,82 & 2,83 & 6,31 \\
Macairea radula (Bonpl.) DC. (Melastomataceae) & 14 & 2,58 & 1,82 & 1,86 & 6,26 \\
Eriotheca gracilipes (K. Schum.) A. Robyns & & & & & \\
(Malvaceae) & 7 & 1,29 & 2,42 & 2,01 & 5,72 \\
Pouteria ramiflora (Mart.) Radlk. (Sapotaceae) & 7 & 1,29 & 2,42 & 1,67 & 5,38 \\
Aspidosperma macrocarpon Mart. (Apocynaceae) & 6 & 1,10 & 1,82 & 2,03 & 4,95 \\
Andira paniculata Benth. (Fabaceae) & 8 & 1,47 & 1,21 & 1,94 & 4,63 \\
& & & & &
\end{tabular}


Qualea multiflora Mart.(Vochysiaceae)

Roupala montana Aubl. (Proteaceae)

Erythroxylum suberosum A. St-Hil.

(Erythroxylaceae)

Ouratea sp. (Ochnaceae)

Pterodon sp. (Fabaceae)

Curatella americana L. (Dilleniaceae)

Annona coriacea Mart. (Annonaceae)

Miconia sp. (Melastomataceae)

Stryphnodendron adstringens (Mart.) Coville

(Fabaceae)

Heteropterys byrsonimifolia A. Juss.

(Malpighiaceae)

Rourea induta Planch. (Connaraceae)

Schefflera macrocarpa (Cham. \& Schltdl.) Frodin

(Araliaceae)

Styrax ferrugineus Nees \& Mart (Styracaceae)

Ferdinandusa elliptica (Pohl.) Pohl (Rubiaceae)

Salacia crassifolia (Mart. ex Schult.) G. Don

(Celastraceae)

Eriotheca pubescens (Mart. \& Zucc.) Schott \&

Endl. (Malvaceae)

Ipomoea pinnata Hochst. Ex Choisy

(Convolvulaceae)

Ouratea spectabilis (Mart.) Engl. (Ochnaceae)

Guapira graciliflora (Mart. ex Schmidt) Lundell

(Nyctaginaceae)

Vochysia elliptica Mart. (Vochysiaceae)

Strychnos pseudoquina A. St.-Hil. (Loganiaceae)

Ficus guaranitica Chodat (Moraceae)

Himatanthus obovatus (Wüll. Arg.) Woodson

(Apocynaceae)

Connarus suberosus Planch. (Connaraceae)

Não Identificada 1

Dimorphandra mollis Benth. (Fabaceae)

Aspidosperma tomentosum Mart. (Apocynaceae)

Diospyros hispida A. DC. (Ebenaceae)

Hancornia speciosa Gomes (Apocynaceae)

Alibertia edulis (Rich.) A.Rich (Rubiaceae)

Palicourea rigida Kunth (Rubiaceae)

Hirtella gracilipes (Hook.f.) Prance

(Chrysobalanaceae)

Anacardium occidentale L. (Anacardiaceae)

Norantea guianensis Aubl. (Marcgraviaceae)

Tocoyena formosa (Cham. \& Schltdl.) K.Schum. $\begin{array}{lllll}5 & 0,92 & 2,42 & 0,76 & 4,10\end{array}$

$5 \quad 0,92 \quad 2,42 \quad 0,44 \quad 3,78$

$6 \quad 1,10 \quad 1,21 \quad 1,45 \quad 3,77$

$\begin{array}{lllll}5 & 0,92 & 1,82 & 0,78 & 3,52\end{array}$

$\begin{array}{lllll}4 & 0,74 & 1,21 & 1,35 & 3,29\end{array}$

$\begin{array}{lllll}3 & 0,55 & 1,82 & 0,83 & 3,20\end{array}$

$\begin{array}{lllll}5 & 0,92 & 1,21 & 1,03 & 3,17\end{array}$

$6 \quad 1,10 \quad 1,21 \quad 0,80 \quad 3,12$

$4 \quad 0,74 \quad 1,21 \quad 1,10 \quad 3,05$

$6 \quad 1,10 \quad 1,21 \quad 0,51 \quad 2,83$

$\begin{array}{lllll}5 & 0,92 & 1,21 & 0,66 & 2,79\end{array}$

$\begin{array}{lllll}4 & 0,74 & 1,21 & 0,77 & 2,72\end{array}$

$\begin{array}{lllll}1 & 0,18 & 0,61 & 1,79 & 2,58\end{array}$

$2 \quad 0,37 \quad 1,21 \quad 0,26 \quad 1,84$

$2 \quad 0,37 \quad 1,21 \quad 0,13 \quad 1,71$

$4 \quad 0,74 \quad 0,61 \quad 0,31 \quad 1,65$

$2 \quad 0,37 \quad 0,61 \quad 0,38 \quad 1,35$

$2 \quad 0,37 \quad 0,61 \quad 0,34 \quad 1,32$

$2 \quad 0,37 \quad 0,61 \quad 0,27 \quad 1,25$

$2 \quad 0,37 \quad 0,61 \quad 0,20 \quad 1,17$

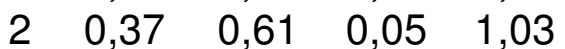

$\begin{array}{lllll}1 & 0,18 & 0,61 & 0,21 & 1,00\end{array}$

$1 \quad 0,18 \quad 0,61 \quad 0,19 \quad 0,98$

$1 \quad 0,18 \quad 0,61 \quad 0,14 \quad 0,93$

$\begin{array}{lllll}1 & 0,18 & 0,61 & 0,14 & 0,93\end{array}$

$\begin{array}{lllll}1 & 0,18 & 0,61 & 0,12 & 0,91\end{array}$

$1 \quad 0,18 \quad 0,61 \quad 0,11 \quad 0,90$

$1 \quad 0,18 \quad 0,61 \quad 0,11 \quad 0,90$

$1 \quad 0,18 \quad 0,61 \quad 0,11 \quad 0,90$

$1 \quad 0,18 \quad 0,61 \quad 0,08 \quad 0,87$

$\begin{array}{lllll}1 & 0,18 & 0,61 & 0,08 & 0,87\end{array}$

$1 \quad 0,18 \quad 0,61 \quad 0,06 \quad 0,85$

$1 \quad 0,18 \quad 0,61 \quad 0,04 \quad 0,83$

$\begin{array}{lllll}1 & 0,18 & 0,61 & 0,04 & 0,83\end{array}$ (Rubiaceae)

\begin{tabular}{rrrrr}
1 & 0,18 & 0,61 & 0,04 & 0,83 \\
\hline 543 & 100 & 100 & 100 & 300
\end{tabular}


Sete espécies representaram 43\% do total das espécies amostradas na área, foram elas: Davilla elliptica (53); Qualea parviflora (43), Sclerolobium paniculatum var subvelutinum (40), Miconia ferruginata (37), Vochysia thyrsoidea (30), Andira paniculata (20) e Byrsonima sp. (23). As dez espécies com maior valor de importância (VI) foram: Q. parviflora, D. elliptica, S. paniculatum, M. ferruginata, Caryocar brasiliense, V. thyrsoidea, A. cujabensis, Qualea grandiflora, Xylopia aromatica e Byrsonima sp., estas contribuíram com cerca de $47,9 \%$ do valor de importância total, representando apenas $17,8 \%$ do total de espécies amostradas (Tabela 2). Além do alto número de indivíduos e de se destacar como o segundo maior VI, D. elliptica teve ocorrência em todas as parcelas, apresentando uma frequência de $100 \%$. M. ferruginata estava distribuída em oito parcelas e $X$. aromatica, $Q$. parviflora, C. brasiliense estavam presentes em sete das 10 parcelas amostradas.

Entre as espécies amostradas 16 apresentaram apenas um indivíduo, correspondendo a $28 \%$ das espécies e juntas representam 2,94\% do número de indivíduos. Foram elas: Alibertia edulis, Connarus suberosus, Diospyros hispida, Ficus guaranitica, Norantea guianensis, Palicourea rigida e Hirtella gracilipes.

As principais famílias encontradas no presente estudo (Fabaceae, Vochysiaceae, Apocynaceae, Malpighiaceae, Melastomataceae e Rubiaceae) são representativas em outras áreas de Cerrado do Brasil Central, sendo Fabaceae a família mais representada neste Domínio (FERNANDES et al., 2013; FINGER; FINGER, 2015; LIMA et al., 2015; GIÁCOMO et al., 2015; SANTOS et al., 2016; FERREIRA et al., 2017). Além das formações savânicas, Fabaceae também é representativa de áreas com formações florestais (FRANCO et al., 2014; SARTORI et al., 2015; SOUZA et al., 2016), também é a principal família encontrada na Mata Atlântica, sendo melhor representada em florestas secas sazonais do que em floresta ombrófila e se destaca, tanto pela diversidade quanto pela riqueza de espécies (GONÇALVES et al., 2016). A maior ocorrência da família Fabaceae é devido a sua capacidade de nodulação em áreas com baixa disponibilidade de nitrogênio e Vochysiaceae é devido à tolerância ao acúmulo de alumínio, duas características marcantes das formações rupestres do Cerrado brasileiro (HARIDASSAN, 2008; RIBEIRO; WALTER, 2008).

As espécies encontradas no presente estudo também foram observadas em outras áreas do Cerrado. No cerrado rupestre da Fazenda Sucupira, DF as mais representativas foram $S$. paniculatum var. subvelutinum, $M$. ferruginata, $Q$. grandiflora (AMARAL et al., 2006). Q. parviflora foi a espécie com maior VI no presente estudo, esta espécie também atingiu os maiores VI na vegetação arbustivo-arbórea em um cerrado rupestre de Cocalzinho de Goiás, GO (PINTO et al., 2009). S. paniculatum var. subvelutinum, obteve o terceiro maior VI no presente estudo e foi a mais representativa na área da Fazenda Sucupira, DF (AMARAL et al., 2006) e na Serra de Caldas Novas, GO (LIMA et al., 2010). Destacando-se também entre as 10 primeiras em VI em diversas áreas de cerrado (MOURA et al., 2007; PINTO et al., 2009).

Foram consideradas espécies raras aquelas com no máximo um indivíduo por hectare. No presente trabalho foram observadas 13 espécies raras: Connarus suberosus, Norantea guianensis e Hirtella gracilipes, sendo que esta não foi encontrada em outros levantamentos de cerrado rupestre e sensu stricto no estado de Goiás (MIRANDA et al., 2007; MOURA et al., 2007; PINTO et al., 2009; LIMA et al., 2010; MOURA et al., 2010; LENZA et al., 2011), mas esteve entre as espécies 
com maiores VI no cerradão na Reserva Mário Viana em Nova Xavantina, MT (MARIMON JÚNIOR; HARIDASAN, 2005) e também em cerrado sensu stricto em Gurupi, TO (FERREIRA et al., 2017). Na área do presente estudo, fragmentos de cerradão e cerrado sensu stricto estão próximas ao local, o que pode explicar a presença desta espécie na área de cerrado rupestre.

$\mathrm{O}$ índice de diversidade de Shannon $\left(\mathrm{H}^{\prime}\right)$ foi igual a 1,51 nats.ind. ${ }^{-1} \mathrm{e} O$ índice de uniformidade de Pielou (J'), igual a 0,37 (Tabela 2). A densidade, o índice de diversidade de Shannon, o índice de uniformidade de Pielou e a área basal das espécies encontradas na área estudada foram baixos quando comparadas com outros estudos em áreas de cerrado rupestre da região Central do Brasil (Tabela 2). Poucos trabalhos relatam densidade e área basal menores que a encontrada nesse trabalho.

Um exemplo de área com densidade e área basal menores é a região dos três picos em Pirenópolis, GO, que apresentou densidade igual a 507 e área basal igual a 3,91 $\mathrm{m}^{2}$ (MOURA et al., 2007) e na Fazenda Sucupira, Brasília, DF que apresentou densidade maior, mas área basal igual a $3,67 \mathrm{~m}^{2}$ (AMARAL et al., 2006) (Tabela 2). Em outras áreas de cerrado rupestre 0 índice de uniformidade varia entre 0,79 e 0,89 (AMARAL et al., 2006; MIRANDA et al., 2007; MOURA et al., 2007; PINTO et al., 2009; LIMA et al., 2010; MOURA et al., 2010; LENZA et al., 2011), enquanto que no presente estudo esse índice foi de 0,37.

Em área de afloramento rochoso no Morro de Bela Vista no Paraná foi observado densidade menor do que a do presente estudo, apenas 256 indivíduos e $H^{\prime}=1,49$ (GILSA et al., 2014), segundo estes autores na área havia apenas três espécies com muitos indivíduos e alto número de espécies com baixa ocorrência. No presente estudo, o baixo índice pode ser explicado pela quantidade de espécies raras, com apenas uma ocorrência, além de ser uma área onde existe grande visitação e foi observada a presença de gado proveniente de fazendas vizinhas 0 que pode estar causando a diminuição das espécies da área.

TABELA 2 - Comparação entre estudos realizados em cerrado rupestre do Brasil Central. 'H' = índice de diversidade de Shannon; J' = índice de uniformidade de Pielou; $A B=$ área basal $\left(m^{2} \cdot h^{-1}\right) ; D A=$ densidade absoluta (ind. ha ${ }^{-1}$ ).

\begin{tabular}{|c|c|c|c|c|c|}
\hline Local & $H^{\prime}$ & $\mathrm{J}$ & $A B$ & DA & Autores \\
\hline Fazenda Sucupira, DF & 3,08 & . & 3,67 & 631 & Amaral et al.(2006) \\
\hline Serra de Caldas Novas, GO & 3,33 & 0,80 & 12,39 & 1.357 & Lima et al. (2010) \\
\hline Serra Dourada, GO & 3,13 & 0,79 & 7,08 & 1.137 & $\begin{array}{l}\text { Miranda et al. } \\
(2007)\end{array}$ \\
\hline $\begin{array}{l}\text { Pirenópolis, GO (Três } \\
\text { Picos) }\end{array}$ & 3,33 & 0,82 & 3,91 & 507 & Moura et al. (2007) \\
\hline Pirenópolis, GO (Portal) & 3,65 & 0,87 & 11,03 & 1.105 & Moura et al. (2010) \\
\hline Alto Paraíso, GO & 3,53 & 0,86 & 7,55 & 893 & Lenza et al. (2011) \\
\hline Cocalzinho de Goiás, GO & 3,45 & 0,83 & 5,67 & 674 & Pinto et al. (2009) \\
\hline $\begin{array}{l}\text { Salto de São Domingos, } \\
\text { Piranhas, GO }\end{array}$ & 1,51 & 0,37 & 4,60 & 543 & Presente estudo \\
\hline
\end{tabular}

A distribuição de diâmetro de todos os indivíduos apresentou um formato "Jinvertido" (Figura 1) sendo que 61,9\% dos indivíduos apresentaram diâmetro máximo de $9,5 \mathrm{~cm}$, havendo poucos indivíduos nas últimas classes. A maioria das 
espécies apresentou altura entre $0,4 \mathrm{~m}$ a três metros sendo que $59,7 \%$ dos indivíduos pertenciam a essas classes (Figura 1). O diâmetro máximo encontrado foi $50,9 \mathrm{~cm}$ para um indivíduo de Emmotum nitens e a altura máxima foi 15 metros para um indivíduo de Pterodon sp. A maioria das espécies apresentou altura e diâmetro pequenos, isto se deve ao fato de que a maioria das espécies do cerrado, principalmente o cerrado rupestre, apresentam árvores baixas e com diâmetros menores (RIBEIRO; WALTER, 2008).
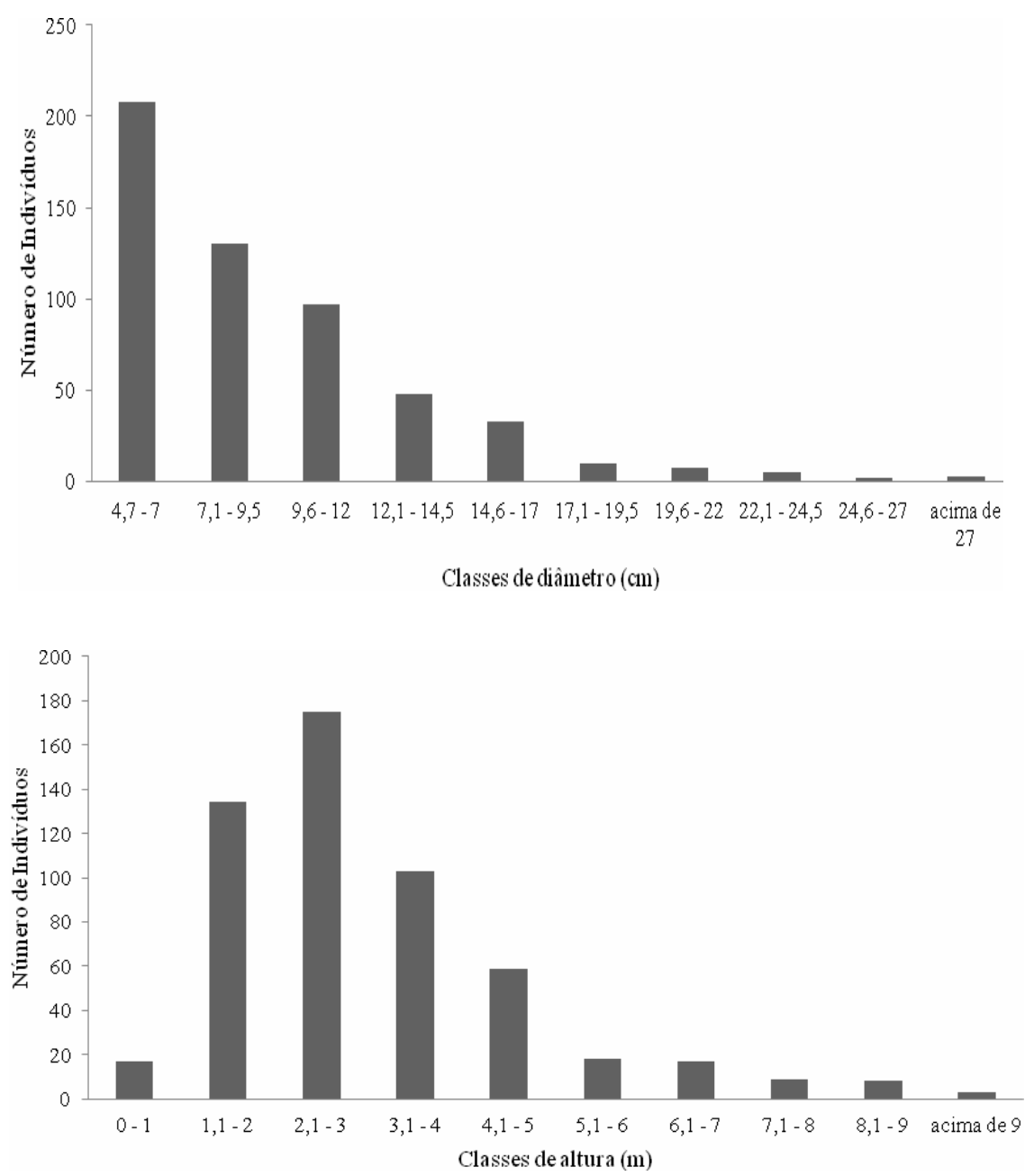

FIGURA 1- Distribuição do número de indivíduos amostrados por classes de diâmetro $(\mathrm{cm})$ e por classes de altura $(\mathrm{m})$ em área de cerrado rupestre no Salto de São Domingos, município de Piranhas, Goiás, Brasil.

\section{CONCLUSÃO}

$\mathrm{Na}$ área do presente estudo foi observada a presença de espécies típicas do cerrado rupestre, no entanto, o baixo valor no índice de diversidade e uniformidade encontrado pode indicar que esta área apresenta grande número de espécies raras (típico de cerrado rupestre), mas que também encontra-se bastante degradada, já que existe uma intensa visitação da população local ao Salto de São Domingos, além da criação de gado que existe na região, sem que haja proteção das espécies nativas presentes no local. 
$\mathrm{Na}$ área foi registrada pela primeira vez no cerrado rupestre do estado de Goiás a espécie Hirtella gracilipes, típica da formação de Cerradão, mostrando a importância da preservação dessa área. Esses índices reforçam a importância de serem realizados mais estudos, além da implantação de programas de educação, manejo e conservação ambiental.

\section{REFERÊNCIAS}

ALVES, R. J. V.; SILVA, N. G.; OLIVEIRA, J. A.; MEDEIROS, D. Circumscribing campo rupestre - megadiverse Brazilian rocky montane savanas. Brazilian Journal of Biology, v. $74, \quad$ n. 2, p. 355-362, 2014. Disponível em: <http://dx.doi.org/10.1590/1519-6984.23212>. doi: 10.1590/1519-6984.23212.

AMARAL, A. G., PEREIRA, F. F.O.; MUNHOZ, C.B.R. Fitossociologia de uma área de cerrado rupestre na Fazenda Sucupira, Brasília, DF. Cerne, v. 12, p. 350-359, $2006 . \quad$ Disponível em: $<$ https://www.agencia.cnptia.embrapa.br/Repositorio/Fitossociologia_Cerrado_Rupes tre_DFID-oRJ8SUQrWH.pdf> Acesso em: 05 abr. 2017.

ANTUNES, K., SALIMENA, F. R. G.; SOBRAL, M. Plinia delicata (Myrtaceae), a new species from southeastern Brazil. Phytotaxa, v. 131, n. 1, p. 45-48, 2013. Disponível em: <http://dx.doi.org/10.11646/phytotaxa.131.1.8>. doi: 10.11646/phytotaxa.131.1.8.

ANGIOSPERM PHYLOGENY GROUP - APG. An update of the Angiosperm Phylogeny Group classification for the orders and families of flowering plants: APG III. Botanical Journal of Linneu Society, v.161, p.105-121. 2009. Disponível em: <http://onlinelibrary.wiley.com/doi/10.1111/j.1095-8339.2009.00996.x/epdf>. Acesso em: 09 dez. 2016..

FELFILI, J.M.; CARVALHO, F.A.; HAIDAR, R.F. Manual para o monitoramento de parcelas permanentes nos biomas cerrado e pantanal. Brasília: Universidade de Brasília, Departamento de Engenharia Florestal, 2005, 55p.

FERREIRA, R. Q. S.; CAMARGO, M. O.; TEIXEIRA, P. R. SOUZA, P. B. SOUZA, D. J. Diversidade florística do estrato arbustivo - arbóreo de três áreas de Cerrado sensu stricto, Tocantins. Revista Desafios, v. 04, n. 02, p. 69-82, 2017. Disponível em: <http://dx.doi.org/10.20873/uft.2359-3652.2017v4n2p69> doi:10.20873/uft.23593652.2017v4n2p69.

FERNANDES, M. H.; ZOCH, V. P.; MATA, R. A.; WALTER, B. M. Fitossociologia do componente arbóreo e florística de um remanescente de cerrado sentido restrito contíguo a áreas de agricultura na porção leste do Distrito Federal, Brasil. Heringeriana, v. 7, n. 1, p. 7-32, 2013. Disponível em; <http://portalinseer.ibict.br/index.php/heringeriana/article/viewFile/1/1>. Acesso em 05 abr. 2017.

FINGER, Z; FINGER, F. A. Fitossociologia em comunidades arbóreas remanescentes de cerrado sensu stricto no brasil central. Floresta, v. 45, n. 4, p. 769-780, 2015. Disponível em: <http://dx.doi.org/10.5380/rf.v45i4.30860>. doi: 
10.5380/rf.v45i4.30860.

FLORA DO BRASIL 2020. Jardim Botânico do Rio de Janeiro. Disponível em:<http://floradobrasil.jbrj.gov.br/ >. Acesso em: 05 Nov. 2016.

FRANCO, B. K. S.; MARTINS, S. V; FARIA, P.C. L.; RIBEIRO, G. A.; NETO, A. M. Estrato de regeneração natural de um trecho de floresta estacional semidecidual, Viçosa, MG. Revista Árvore, v. 38, n. 1, p. 31-40, 2014. Disponível em: <http://dx.doi.org/10.1590/S0100-67622014000100003> doi: 10.1590/S010067622014000100003.

FREITAS, H. S.; BURSTIN, B.; FERREIRA, G.; ALEGRETTI, L.; FLYNN, M. Levantamento florístico na Estação Experimental da Syngenta em Uberlândia. Revista Intertox de Toxicologia Risco Ambiental e Sociedade, v. 9, n. 1, p. 3669, $2016 . \quad$ Disponível em:

file:///C:/Users/vsard 000/Documents/Artigos/Flor\%C3\%ADstica,\%20Fitossociologia \%20e\%20Estrutura/Freitas\%20et\%20al,\%202016.pdf>.Acesso em 05 abr.2017.

GIÁCOMO, A. G., PEREIRA, M. G.; CARVALHO, D.C; MEDEIROS, V.S.; GAUI, T.D. Florística e Fitossociologia em Áreas de Cerradão e Mata Mesofítica na Estação Ecológica de Pirapitinga, MG. Floresta e Ambiente, v. 22, n. 3, p. 287-298, 2015. Disponível em: <http://dx.doi.org/10.1590/2179-8087.067913> doi: 10.1590/21798087.067913.

GILSA, E. A. V.; HOMCZINSKI, I. KRUPEK, R. A. Levantamento florístico e fitossociológico em uma área do Morro Bela Vista, no município de Porto União SC. Ambiência, v. 10, n. 2, p.581-596, 2014. Disponível em: <http://dx.doi.org/10.5935/ambiencia.2014.02.11 >doi:10.5935/ambiencia.2014.02.11.

GOMES, L; MARACAHIPES, L.; REIS, S. M.; MARIMON, B. S; MARIMON-JUNIOR, B.H.; LENZA, E. Dynamics of the woody vegetation of two areas of Cerrado sensu stricto located on different substrates. Rodriguésia, v. 67, n. 4, p. 859-870, 2016. Disponível em: <http://dx.doi.org/10.1590/2175-7860201667401> doi: 10.1590/21757860201667401.

GONÇALVES, T. S; SILVA, R. H. H.; SOUZA, S. R.; VELOSO, M. D. M.; NUNES, Y. R. F. A vegetação dos afloramentos calcários na Serra do Cipó. Revista Espinhaço, v. $5, \quad$ n. 1, p. 19-31. 2016. Disponível em: <http://www.revistaespinhaco.com/index.php/journal/article/view/97>. Acesso em: 02 out. 2017.

HARIDASAN,, M. Nutritional adaptations of native plants of the cerrado biome in acid soils. Revista Brasileira de Fisiologia Vegetal, v. 20, n. 3, p. 183-195. 2008. Disponível em: http://www.scielo.br/pdf/bjpp/v20n3/a03v20n3.pdf. Acesso em: 01/10/2017.

IBGE - Instituto Brasileiro de Geografia e Estatística, Cidades, Censo 2010. Disponível em:<http://www.ibge.gov.br/cidadesat/ topwindow.htm?1>. Acesso em: 25 mar. 2017. 
LENZA, E; PINTO, J. R. R.; PINTP, A. S.; MARACAHIPES, L.; BRUZIGUESSI, E. P. Comparação da vegetação arbustivo-arbórea de uma área de cerrado rupestre na Chapada dos Veadeiros, Goiás, e áreas de cerrado sentido restrito do Bioma Cerrado. Revista Brasileira de Botânica, v.34, n.3, p. 247-259, 2011. Disponível em: <http://www.scielo.br/pdf/rbb/v34n3/02.pdf> Acesso em: 05 abr. 2017.

LIMA, T. A.; PINTO, J. R. R.; LENZA, E.; PINTO, A. S. Florística e estrutura da vegetação arbustivo-arbórea em uma área de cerrado rupestre no Parque Estadual da Serra de Caldas Novas, Goiás. Biota Neotropica, v. 10, n. 2, p. 159-166, 2010. Disponível em: <http://dx.doi.org/10.1590/S1676-06032010000200020> doi: 10.1590/S1676-06032010000200020.

LIMA' R. A. F., RANDO, J. G. BARRETO, K. D. Composição e diversidade no cerrado do Leste de Mato Grosso do Sul, Brasil. Revista Árvore, v. 39, n. 1, p. 9-24 2015. Disponível em: <http://dx.doi.org/10.1590/0100-67622015000100002> doi: 10.1590/0100-67622015000100002.

MAGURRAN, A. E. Ecological diversity and its measuremnts. London: Croom Hell Limited. 1988, 179p.

MARIMON JUNIOR, B. H.; HARIDASAN, M. Comparação da vegetação arbórea e características edáficas de um cerradão e um cerrado sensu stricto em áreas adjacentes sobre solo distrófico no leste de Mato Grosso, Brasil. Acta Botanica Brasilica, v.19, n.4, p. 913-926, 2005. Disponível em: <http://www.scielo.br/pdf/abb/v19n4/a26v19n4.pdf> Acesso em 05 abr. 2017.

MOTA, S. L. L., PEREIRA, I. M., MACHADO, E. L. M., OLIVEIRA, M. L. R., BRUZINGA, J. S., FARNEZI, M. M. M., \& JUNIOR, M. S. M. Influência dos Afloramentos Rochosos sobre a Comunidade Lenhosa no Cerrado stricto sensu. Floresta e Ambiente, v. 21, n. 1, p. 8-18, 2014. Disponível em: <http://dx.doi.or/ 10.4322/floram.2014.009> doi: 10.4322/floram.2014.009.

MELO, P. H. A.; LOMBARDI, J. A.; SALINO, A.; CARVALHO, D. A. Composição florística de angiospermas no carste do Alto São Francisco, Minas Gerais, Brasil. Rodriguésia, v. 64, n..1, p. 29-36, 2013. Disponível em: <http://dx.doi.org/10.1590/S2175-78602013000100004> doi: 10.1590/S217578602013000100004.

MIRANDA, S. C., SILVA JÚNIOR, M. C., SALLES, L. A. A comunidade lenhosa de cerrado rupestre na Serra Dourada, Goiás. Heringeriana, v. 1, n. 1. p. 43-53, 2007. Disponível em: < http://portalinseer.ibict.br/index.php/heringeriana/article/view/117> Acesso em: 05 abr. 2017.

MOURA, I. O.; GOMES-KLEIN, V. L.; FELFILI, J. M.; FERREIRA, F. D. Fitossociologia da comunidade lenhosa de uma área de cerrado rupestre no Parque Estadual dos Pireneus, Pirenópolis, Goiás. Revista de Biologia Neotropical, v.4, p. 83-100, 2007. Disponível em: <https://www.revistas.ufg.br/RBN/article/view/5208> Acesso em: 05 abr. 2017. 
MOURA, I. O.; GOMES-KLEIN, V. L.; FELFILI, J. M.; FERREIRA, F. D. Diversidade e estrutura comunitária de cerrado sensu stricto em afloramentos rochosos no Parque Estadual de Pireneus, Goiás. Revista Brasileira de Botânica, v. 33, n. 3, p. 455-467, 2010. Disponível em: <http://dx.doi.org/10.1590/S010084042010000300008> doi: 10.1590/S0100-84042010000300008.

MUELLER-DOMBOIS, D.; ELLENBERG, H. Aims and methods of vegetation ecology. John Wiley and Sons, New York, 1974, 547p.

PIELOU, E.C. Ecological diversity. New York: John Wiley \& Sons, 1974, 547p.

PINTO, J. R. R.; LENZA, E.; PINTO, A. S. Composição florística e estrutura da vegetação arbustivo-arbórea em um cerrado rupestre, Cocalzinho de Goiás, Goiás. Revista Brasileira de Botânica, v. 32, n.1, p. 1-10, 2009. Disponível em: <http://dx.doi.org/10.1590/S0100-84042009000100002> doi: 10.1590/S010084042009000100002.

RIBEIRO, J. F.; WALTER, B. M. T. As principais fitofisionomias do bioma Cerrado. In: Cerrado: ecologia e flora. Planaltina: EMBRAPA. 2008, p. 151-212.

SANTOS, M.G.F.N. O município de Piranhas: geografia, história e educação ambiental. Editora Kelps, 2001, 161p.

SANTOS, L. R. T.; FERREIRA, H. D.; FARIA, M. T. Levantamento florístico da comunidade arbóreo-arbustiva, do Parque Sulivan Silvestre Goiânia, Go, margenado a nascente do Córrego Vaca-Brava. Revista Eletrônica de Educação da Faculdade Araguaia, v. 9, p. 38-48, 2016. Disponível em: <http://www.fara.edu.br/sipe/index.php/renefara/article/view/418> Acesso em: 02 de out. 2017.

SARTORI, R. A.; CARVALHO, D. A. VAN DEN BERG, E.; MARQUES, J. J. G. S. M; SANTOS, R. M. Variações florísticas e estruturais do componente arbóreo de uma floresta estacional semidecidual montana em Socorro, SP. Rodriguésia, v. 66, n.1, p. 33-49, 2015. Disponível em: <http://dx.doi.org/10.1590/2175-7860201566103> doi: $10.1590 / 2175-7860201566103$.

SILVA, J. B. Panorama sobre a vegetação em afloramentos rochosos do Brasil Oecologia Australis, v. 20, n. 4, p. 451-463, 2016. Disponível em <http://dx.doi.org/10.4257/oeco.2016.2004.05> doi: 10.4257/oeco.2016.2004.05.

SILVA, J. B.; GERMANO, S. R. Brioflora de Afloramentos Rochosos: uma visão conservacionista. Acta Botanica Brasilica, v. 27, n. 4, p. 827-835, 2013. Disponível em: <http://dx.doi.org/10.1590/S0102-33062013000400023> doi: 10.1590/S010233062013000400023.

SILVEIRA, F.A.O., NEGREIROS, D., BARBOSA, N.P.U. et al. Ecology and evolution of plant diversity in the endangered campo rupestre: a neglected conservation priority. Plant Soil, v. 403, n. 1, p. 129-152, 2016. Disponível em: 
<http://dx. doi.org/10.1007/s11104-015-2637-8> doi: 10.1007/s11104-015-2637-8.

SOUZA, K. R.; OLIVEIRA, G M.; FERREIRA, F.G.; MARTINS, T. O.; SILVA NETO, C.M.; VENTUROLI, F. Floresta Estacional: madeira para o produtor rural no Cerrado. Magistra, v. 28, n.1, p.54-62, 2016. Disponível em: $<$ https://magistraonline.ufrb.edu.br/index.php/magistra/article/view/71/232> Acesso em: Acesso em: 02 de out. 2017.

TORRES, D. M.; FONTES, M. A. L.; SAMSONAS, M H. P. Relações solo-vegetação na estruturação de comunidades de cerrado sensu stricto no sul de Minas Gerais, Brasil. Rodriguésia, v. 68, n. 1, p.115-128, 2017. Disponível em: <http://dx.doi.org/10.1590/2175-7860201768121> doi: 10.1590/21757860201768121.

VERÇOZA, F. C.; BASTOS, M. S. Bromeliaceae e Cactaceae dos afloramentos rochosos do Costão de Itacoatiara, Parque Estadual da Serra da Tiririca, Niterói, Rio de Janeiro, Brasil. Natureza on line, v. 11, n. 1, p. 7-11, 2013. Disponível em <http://www.naturezaonline.com.br/natureza/conteudo/pdf/02_Ver\%C3\%A7oza\&Bast osl_007011.pdf> Acesso em 07 abr. 2017. 